\title{
LA OPINIÓN NEGATIVA DEL SÍ-MISMO EN LOS TRASTORNOS DEPRESIVOS
}

\author{
AMPARO GIMÉNEZ y AMPARO BELLOCH
}

Facultad de Psicología, Universidad de Valencia

(Aceptado en enero de 2002)

\begin{abstract}
Las teorfas cognitivas de la depresión (Beck, 1967, 1976) señalan que los sesgos negativos en el procesamiento de información son los responsables del mantenimiento, gravedad y duración del estado de ánimo deprimido. Sin embargo, los resultados de los estudios que intentan identificar tales sesgos no son consistentes. En este contexto, nuestro trabajo plantea explorar si durante un episodio depresivo el paciente sigue teniendo acceso a información tanto positiva como negativa sobre sí mismo, aunque matizada por criterios temporales. Para ello, 20 pacientes con depresión mayor, 18 distímicos y 20 sujetos controles no psiquiátricos completaron la tarea de codificación autorreferente, manipulando las instrucciones para que la realizaran teniendo en cuenta tres referentes temporales distintos: presente (actual), pasado y futuro. Los resultados mostraron que los deprimidos tienen una visión diferente de sí mismos para el presente, el pasado y el futuro, con matices interesantes por sus implicaciones en la investigación y en la clínica. Además, se observó que los deprimidos tienen una visión más negativa de sí mismos que los controles no deprimidos, independientemente del referente temporal que se utilice. Se discuten las repercusiones que estos resultados pueden tener tanto a nivel clínico como en la investigación de la psicopatología depresiva.
\end{abstract}

Palabras clave : Depresión, sí-mismo, auto-esquema, auto-percepción, rasgo-estado.

\section{Negative view about the self in depressive disorders}

According to cognitive theory of depression (Beck, 1967, 1976), negatively biased information processing is responsible for maintenance, severity and duration of depressive affect. However, the evidence does not consistently support this assumption. The present study was designed to ascertain if depressed patients have access not only to negative information about the self, but also to positive one. Twenty patients with major depressive disorder, 18 with distimic disorder, and 20 nonpsychiatric controls completed a self-referent task. Instructions to perform the task were manipulated in order to fulfill it taking into account three different time-periods: present, past, and future. Data showed that depressed patients had a different self-perception on each of the three time-periods investigated. Moreover, we observed that they had a more negative self-perception than nonpsychiatric controls during all of the time-periods. Implications for research and clinical practice are suggested.

Key words: Depression, self-view, self-schema, self-perception, trait-state.

\section{INTRODUCCIÓN}

La depresión es un trastorno que sigue despertando el interés de los investigadores que se sitúan en la perspectiva del procesamiento de información (Ingram

Correspondencia: Amparo Belloch, Dpto. de Personalidad, Evaluación y Tratamiento Psicológicos, Facultad de Psicologia, Av. Blasco lbáñez 21, 46010 Valencia. Correo-e: Amparo.Belloch@uv.es, Fax (+34) 963864669 et al., 1998; Clark et al., 1999; Dozois y Dobson, 2001). En este contexto, los distintos modelos explicativos que se han generado sobre el trastorno destacan el papel que tienen los sesgos cognitivos en la etiología, mantenimiento, gravedad y duración del estado de ánimo depresivo. En todo caso, el planteamiento dominante desde hace años para la comprensión de la depresión unipolar sigue siendo el de A.T. Beck (Beck et al., 1979; 
Salkovskis, 1996). De acuerdo con este modelo, los individuos que experimentan pérdidas o adversidades en la infancia desarrollan esquemas negativos (esquemas depresógenos) relacionados con pérdida, fracaso o abandono. Dichos esquemas se reactivan cuando el individuo experimenta posteriormente un suceso vital que guarda semejanza con los sucesos adversos iniciales, y actúan como filtros a través de los cuales los estímulos y los sucesos del entorno son percibidos, atendidos, evaluados y recordados. En consecuencia, estos filtros actúan como sesgos, es decir, tendencias preferentes o pre-juiciosas, que favorecen el procesamiento de la información congruente con el contenido de los esquemas. Por lo tanto, en el modelo de Beck los sesgos de procesamiento refuerzan, por un lado, la actividad de los esquemas depresógenos, y por otro lado explican la persistencia de los síntomas depresivos. Bower (1981) y Teasdale $(1983,1988)$ articularon posteriormente modelos cognitivos similares. En ambos casos, se enfatiza también el impacto que tienen los sesgos cognitivos en el mantenimiento, gravedad y duración del estado de ánimo depresivo.

La investigación empírica sobre la depresión unipolar se ha centrado tanto en los productos del sistema cognitivo (Mc Leod y Hagan, 1992) como en sus operaciones (Haaga et al., 1991; Clark y Steer, 1996; Clark et al., 1999). Más recientemente algunos investigadores han intentado además evaluar la estructura cognitiva (Segal y Gemar, 1997). Inicialmente, los resultados de este tipo de investigaciones parecían corroborar algunas de las hipótesis derivadas del modelo de Beck. Por ejemplo, los trabajos de Derry y Kuiper (1981), Clark y Teasdale (1985), o en nuestro contexto, Ruipérez (1994), evidenciaron la presencia de sesgos en el procesamiento de la informacion de los deprimidos, destacando la presencia de un recuerdo aumentado de la información autorreferente negativa en estos pacientes, en comparación con personas no deprimidas. El meta-análisis realizado por Matt, Vázquez y Campbell (1992), avalo estos resultados para los estudios con poblaciones clínicas. Sin embargo, estudios posteriores no han logrado reproducir ese mismo efecto, utilizando diseños experimentales similares, e igualmente muestras clínicas (Hedlund y Rude, 1995, Calev, 1996, Giménez, 2000). A la vista de estos resultados, la evidencia de un sesgo de memoria en el procesamiento de información autorreferente en personas deprimidas, considerando especialmente el recuerdo incidental, no se ha mostrado consistente.

Para dar cuenta de tales discrepancias algunos investigadores han revisado el supuesto de que el contenido de la información almacenada en el autoesquema sea monolítica y en gran parte estable. Más bien al contrario: sugieren que la información que contiene el autoesquema sobre el sí-mismo, hace referencia a características estables de la personalidad, pero también a auto-valoraciones actuales, que en parte se hallan moduladas por el estado de ánimo en el que uno se encuentra (Hammen et al., 1985; Dohr et al., 1989). Si esto fuera así, es probable que surgieran discrepancias entre los contenidos mismos del autoesquema, y que éstas fueran responsables de los resultados inconsistentes que se obtienen cuando se pide a una persona que se auto-describa. Esta sugerencia es, en nuestra opinión, coherente con la observación clínica, al menos en el caso de muchos deprimidos: estos pacientes tienen una auto-percepción negativa de sí mismos en el "aquí-y-ahora», pero al mismo tiempo son capaces de manifestar aspectos positivos de sí mismos en el pasado (antes de la depresión), a la vez que esperan "reencontrarse» en un estado más positivo en el futuro. 
De hecho, otros autores (Ingram y Reed, 1986; Chaplin et al., 1988; Brown et al., 1990; Ingram et al., 1995) han discrepado de la idea de un autoesquema depresivo monolítico, sugiriendo que éste posee información tanto positiva como negativa. Consideran que para aproximarse mejor a las representaciones mentales que los deprimidos tienen sobre ellos mismos, podría ser útil: a) conocer si la información a la que tienen acceso durante un episodio depresivo se experimenta como relacionada con una personalidad estable (rasgo) o, por el contrario, con su estado actual (estado); y, b) saber si estos sujetos continúan teniendo acceso tanto a la información positiva como a la negativa sobre el sí mismo.

La investigación revela de forma consistente que los sujetos deprimidos, en comparación con los no deprimidos, identifican más atributos negativos como autodescriptores (Derry y Kuiper, 1981; Ruipérez, 1994; Giménez, 2000). Ahora bien: ¿este efecto es consistente cuando se introducen marcadores temporales en la autodescripción?. Es decir, si se les pide a los deprimidos que se auto-describan no solo en el momento presente, sino también en el anterior a su estado depresivo ¿seguirán auto-identificándose con atributos negativos en su pasado reciente?

Considerando que las referencias de trabajos que abordan este tema son todavía escasas y el interés que sus propuestas pueden tener tanto a nivel experimental como clínico, decidimos elaborar un estudio para intentar responder a estos interrogantes. Por un lado, pretendemos averiguar si durante un episodio depresivo los sujetos se perciben a sí mismos en términos enteramente negativos, o si reconocen aspectos positivos y negativos de sí mismos en función del referente temporal en el que se sitúen (presente, pasado y futuro). Por otro, nos interesa examinar las diferencias entre la visión que tienen de sí mismos estos pacientes y la de personas no deprimidas, tomando también en consideración los tres referentes temporales mencionados. Nuestros objetivos se concretan como sigue: (1) Analizar si el autoesquema actual de los pacientes deprimidos es predominantemente negativo, en comparación con la visión de sí mismos en el pasado y el futuro, que se predice compuesta igualmente de elementos positivos y negativos; y (2) Averiguar si los pacientes deprimidos muestran un autoesquema actual diferente al de los sujetos no deprimidos, mientras que la visión de sí mismos en el pasado y el futuro es similar y está compuesta de elementos positivos y negativos de forma proporcionada.

Con el fin de abordar el estudio del autoesquema, son muchos los investigadores que han utilizado la tarea de codificación autorreferente (TCA) (Craik y Lockhart, 1972). La tarea consiste en presentar consecutivamente a los sujetos un número predeterminado de adjetivos que describen aspectos personales y que difieren en la valencia afectiva, para que decidan categorialmente (Sí o No) si son autodescriptores. Posteriormente se realiza una prueba de recuerdo incidental. Las medidas (variables dependientes) de esta tarea incluyen el número y valencia de los adjetivos escogidos como autodescriptores, el tiempo requerido para emitir el juicio, y la tasa de recuerdo para los adjetivos presentados. De este modo, la TCA proporciona distintas medidas que reflejan el funcionamiento de los esquemas subyacentes (Segal y Swallow, 1994). En particular, el número de adjetivos positivos y negativos que el individuo escoge como autodescriptores, proporciona un índice de la tasa relativa de información positiva y negativa sobre el sí mismo a la que el sujeto tiene mayor accesibilidad.

Se han empleado dos estrategias experimentales distintas para investigar las 
diferencias entre rasgo-estado en cuanto al contenido del autoesquema: algunos autores (Ingram et al., 1994, 1995), manipulan la selección del material estimular, ampliando las categorías empleadas hasta el momento. En vez de incluir únicamente adjetivos con valencia emocional negativa y no negativa, catalogan el material en adjetivos negativos-rasgo, negativos-estado y no negativos. Otros trabajos (Brewin et al., 1992) utilizan otra estrategia que consiste en manipular únicamente las instrucciones experimentales para que los sujetos procesen el material estimular en función de distintos momentos temporales (por ejemplo, actual y en general). Nuestro trabajo utiliza este segundo procedimiento.

\section{MÉTODO}

\section{Participantes}

Se utilizó una muestra clínica de pacientes deprimidos y una muestra control, con ausencia de depresión. Se contemplaron los siguientes criterios de selección generales, para todos los sujetos: (1) edad comprendida entre 18 y 65 años, (2) ausencia de historia de alcoholismo o adicción a drogas, (3) ausencia actual de enfermedad orgánica grave, (4) ausencia de dificultades para leer. Para la muestra clínica se aplicó como criterio adicional la ausencia actual de otros trastornos psicológicos asociados. Para la muestra control se exigieron dos criterios adicionales, usencia de antecedentes personales de trastornos afectivos, y ausencia de sintomatología depresiva actual.

El grupo clínico se seleccionó de forma consecutiva entre los pacientes externos que acudían a la Policlínica de Psiquiatría del Hospital Clínico de Valencia, y satisfacian los criterios diagnósticos DSM-IV (APA, 1994) para tras- torno depresivo mayor o trastorno distímico. Todos ellos fueron evaluados por la primera autora mediante la entrevista estructurada SCID (First et al., 1997) El grupo control se seleccionó una vez se hubo completado la muestra clínica entre personas que se ajustaran a las mismas características socio-demográficas de los pacientes.

La muestra definitiva estuvo compuesta por 20 personas diagnosticadas de trastorno depresivo mayor ( 3 hombres y 17 mujeres; media de edad de 42,65 años), 18 sujetos diagnosticados de trastorno distímico ( 2 hombres y 16 mujeres; media de edad de 40 años) y 20 sujetos controles ( 6 hombres y 14 mujeres, con media de edad de 39,45 años). En la Tabla 1 se recogen los valores descriptivos de cada una de las variables demográficas analizadas. Como se puede constatar, no hubo diferencias entre los tres grupos en ninguna de ellas.

\section{Instrumentos y procedimiento}

Utilizamos la tarea de codificación autorreferente (TCA), antes descrita. La TCA se administró en la tres condiciones siguientes, que variaban en cuanto a las instrucciones experimentales: (1) Valoración de sí mismo en el presente (actual): "A continuación van a aparecer en la pantalla palabras que sirven para describir a las personas. Quiero que tome como referencia cómo se ve usted a sí mismo en estos últimos días, incluido el día de hoy. Si esas palabras le describen a usted actualmente, apretará la tecla del SI, y si no le describen a usted actualmente, apretará la tecla del NO». (2) Valoración de sí mismo en el pasado (pasado): «Vamos a realizar una prueba muy parecida a la anterior. Van a aparecer en la pantalla palabras que sirven para describir a las personas. Quiero que usted me diga si esas palabras le describen a usted 
Tabla 1. Descripción de variables sociodemográficas (datos en frecuencias) y contrastes entre los grupos

\begin{tabular}{|c|c|c|c|c|c|}
\hline \multirow[b]{2}{*}{ Variable } & \multirow[b]{2}{*}{ Categorías } & \multicolumn{3}{|c|}{ Diagnóstico } & \multirow[b]{2}{*}{ Contrastes } \\
\hline & & Depresion mayor & Distimia & Control & \\
\hline \multirow[t]{2}{*}{$\overline{\text { Sexo }}$} & Hombre & 3 & 2 & 6 & $2,51^{2} n s$ \\
\hline & Mujer & 17 & 16 & 14 & \\
\hline \multirow[t]{3}{*}{ Edad } & $18-34$ & 6 & 5 & 11 & $0,33^{1} n s$ \\
\hline & $35-50$ & 7 & 9 & 4 & \\
\hline & $51-65$ & 7 & 4 & 5 & \\
\hline \multirow{4}{*}{$\begin{array}{l}\text { Estado } \\
\text { civil }\end{array}$} & Soltero & .4 & 3 & 8 & $1,93^{2} n s$ \\
\hline & Viudo & 1 & 0 & 0 & \\
\hline & Separado & 1 & $\mathbf{0}$ & 1 & \\
\hline & Casado & 14 & 15 & 11 & \\
\hline \multirow{4}{*}{$\begin{array}{l}\text { Nivel } \\
\text { educativo }\end{array}$} & Primaria incom. & 3 & 2 & 7 & $0,54^{2} n s$ \\
\hline & Primaria comp. & 8 & 8 & 5 & \\
\hline & Medios & 8 & 6 & 4 & \\
\hline & Superiores & 1 & 2 & 4 & \\
\hline \multirow{5}{*}{$\begin{array}{l}\text { Situación } \\
\text { Laboral }\end{array}$} & Activo & 4 & 7 & 12 & $0,31^{2} n s$ \\
\hline & Est./ A. de casa & 2 & 1 & 5 & \\
\hline & Baja & 11 & 6 & 1 & \\
\hline & Pensionista & 0 & 1 & 1 & \\
\hline & Parado & 3 & 3 & 1 & \\
\hline \multirow{5}{*}{ Económico } & Bajo & 7 & 2 & $\mathbf{0}$ & $2,06^{3} n s$ \\
\hline & Medio-bajo & 4 & 4 & 7 & \\
\hline & Medio & 6 & 11 & 13 & \\
\hline & Medio-alto & 3 & 1 & 0 & \\
\hline & Alto & $\mathbf{0}$ & 0 & 0 & \\
\hline
\end{tabular}

Nota: ${ }^{1}$ ANOVA, ${ }^{2} \chi^{2}$ (prueba de la mediana), ${ }^{3} \mathrm{H}$ de Kruskal-Wallis.

en general, su forma de ser de siempre, antes de aparecer la depresión. Si describen su forma de ser antes de estar deprimido/a, apretará la tecla del SI, y si no es así, apretará la tecla del NO». Y (3) valoración de sí mismo en el futuro (futuro): "Igual que en las pruebas anteriores, aparecerán en la pantalla palabras que sirven para describir a las personas. Lo que quiero que me diga ahora es cuáles de esas palabras piensa que le describirán a usted en el futuro y cuáles no. Es decir, si piensa que una palabra le describirá en el futuro, apretará la tecla del SI, y si cree que no le describirá, apretará la tecla del NO. Es importante que decida según como crea que se verá realmente en el futuro, y no tanto en función de cómo se desearía ver".

La selección de los estímulos se realizó a partir de un estudio normativo pre- vio. Tomando como referencia el trabajo de Ruipérez (1994) se elaboró un banco inicial de palabras que fue sometido a una evaluación interjueces, quienes establecían la pertenencia al campo semántico y el grado de dificultad de los estímulos. Finalmente se seleccionó un total de 30 palabras, 10 con valencia negativa-depresiva, 10 con valencia positiva-no depresiva, y 10 neutras desde el punto de vista del estado de ánimo (Anexo 1). Es importante resaltar que los descriptores se escogieron para identificar estados de ánimo positivos y negativos, y no características de personalidad (rasgos). Estas se relegaron al apartado de neutros, e incluían tanto características más bien consideradas en general como positivas (p.ej., comprensivo), o por el contrario como negativas (p. ej., agresivo), o como neutras (p.ej., presu- 
mido). En definitiva, el listado de «neutros» hace referencia a neutralidad en cuanto a estado de ánimo. Asimismo se constató que la longitud (sílabas y letras) de palabras asignadas a cada categoría se mantuviera balanceada.

Para la presentación de los estímulos utilizamos un ordenador personal PC 486. En la pantalla aparecían uno por uno los estímulos, y una rutina del ordenador aleatorizaba el orden de aparición en cada presentación. El tiempo de exposición no tenía límite, apareciendo una nueva palabra estímulo en el momento en que el sujeto dejaba de pulsar la tecla «SI» o «NO». Se utilizó un teclado expandido, y para facilitar la tarea, el "NO» se colocó en la barra espaciadora del bloque alfanumérico y el "SI" en las teclas de dirección del bloque de control del teclado. En la primera presentación, se realizaba una tarea de ensayo con objeto de que los sujetos se familiarizasen con las teclas y con la aparición de los estímulos. En dicha tarea se pedía a los sujetos que indicaran si las palabras que aparecían en la pantalla correspondían o no a "comida». Una vez comprobado que los sujetos habían comprendido la mecánica de la prueba y, en su caso, corregidos los errores y/o las dudas que pudieran surgir, se procedía a la realización de la TCA propiamente dicha, en el orden siguiente: condición actual, pasado, futuro. Para cada caso se dieron las instrucciones correspondientes que se han relatado más arriba. Todos los sujetos dieron su consentimiento expreso para participar en la investigación.

\section{Análisis estadísticos}

Los datos de este estudio se analizaron con el programa estadístico SPSS/PC+ 9.0. Previamente a la aplicación de pruebas estadísticas se comprobaron los supuestos de normalidad y de homogeneidad de varianzas. Además, en el caso de los ANOVAs de medidas repetidas, se comprobó el supuesto de esfericidad, y en caso de incumplimiento del mismo, el valor considerado fue la Traza de Pillai, una aproximación al valor de $F$. Los datos se expresan como medias (entre paréntesis se indican las desviaciones típicas). El valor mínimo exigido de significación estadística fue $p<0.05$.

\section{RESULTADOS}

Objetivo primero: Autoesquema actual negativo en los pacientes deprimidos

Se consideraron los dos grupos clínicos (depresión mayor y distimia). Para cada uno se realizaron tres ANOVAs, uno para cada condición, de medidas repetidas de un factor, con tres niveles: adjetivos positivos, adjetivos negativos y adjetivos neutros.En primer lugar se analizaron los resultados obtenidos intra-grupo/intracondición. Los pacientes con depresión mayor mostraron los siguientes resultados. Presente: $F_{(2,18)}=243,71 ; p<0,001$; Pasado: $F_{(2,18)}=7,324, p<0,005$; Futuro: $F_{(2,18)}=2,74 ; p<0,091$. Por su parte, en los distímicos se obtuvieron estos resultados: Presente: $F_{(2,16)}=16,23, p<0,001$; Pasado: $F_{(2,16)}=2,44, p<0,119$; Futuro: $F_{(2,16)}=0,029 ; p<0,971$.

Con el fin de identificar la fuente de las diferencias aparecidas en algunos de los casos, realizamos contrastes específicos (Helmert y Difference), comparando las medias de los adjetivos autoadscritos, según su valencia, dentro de cada condición. Los resultados mostraron que el grupo de depresión mayor en la condición Actual, escogí más autodescriptores negativos $(8,7(1,59))$ que positivos $(0,2(0,41))$ y neutros $(5,05(1,63))$, y más neutros que positivos. En el caso de la condición Pasado escogió más autodes- 
criptores positivos $(7,55(2,85))$ y neutros $(6,25(1,29))$ que negativos $(3,4(2,77))$. $Y$ para la condición Futuro, no se encontraron diferencias significativas en la valencia de los adjetivos que se autoadscribieron. El grupo de distimia escogió, en la condición Actual, más autodescriptores negativos $(7,38(2,52))$ y neutros $(5,77(1,62))$ que positivos $(2,5(2,43))$. $\mathrm{Y}$ para las condiciones Pasado y Futuro no se encontraron diferencias significativas..

El segundo análisis intra-grupos y entre-condiciones, se realizó mediante tres ANOVAs de medidas repetidas (uno para cada valencia) de un factor, con tres niveles: condición Actual, condición Pasado y condición Futuro. El grupo de depresión mayor presentó los siguientes resultados: Negativas. $F_{(2,18)}=26,69, p<$ 0,001; Positivas: $F_{(2,18)}=52,89, p<0,001$; Neutras: $F_{(2,18)}=6,72, p<0,001$. Y el grupo de Distimia obtuvo: Negativas: $F_{(2,16)}=$ 6,49, $p<0,001$; Positivas: $F_{(2,16)}=11,13, p$ $<0,001$; Neutras: $F_{(2,16)}=0,37$ (n.s.). Los contrastes específicos mostraron que los pacientes con depresión mayor escogieron más autodescriptores negativos en la condición Actual $[8,7(1,59)]$ que en el Pasado $[3,40(2,77)]$ y el Futuro $[4,45$ $(3,26)]$, y más en el Futuro que en el Pasado. Para los rasgos positivos mostraron el patrón inverso, escogiendo menos para la condición Actual $[0,2(0,471)]$ que para el Pasado $[7,55(2,85)]$ y el Futuro $[6,1$ $(3,86)]$, y menos para el Futuro que para el Pasado. En cuanto a los distímicos, presentaron idéntico patrón que los pacientes con depresión mayor para los rasgos negativos, escogiendo más para la condición Actual [7,38 (2,52)] que para el Pasado $[3,77(3,17)]$ y el Futuro $[5,61$ $(3,63)]$, y más para el Futuro que para el Pasado. Y similar para los rasgos positivos, escogiendo menos en la condición Actual $[2,5(2,43)]$ que para el Pasado $[6,72(3,57)]$ y el Futuro [6(3,59)], pero sin haber diferencias entre estas dos últimas condiciones.
Objetivo segundo: Diferencias en el autoesquema actual entre deprimidos y no deprimidos

Empleamos los tres grupos incluidos en el estudio. El ANOVA de medidas repetidas mixto $9 \times 3$ (valencia según condición $\times$ grupo) mostró diferencias estadísticamente significativas para el efecto Valencia $\left(F_{(8,48)}=25,17 ; p<0,001\right)$ y para la interacción $\left(F_{(16,98)}=8,27, p<\right.$ 0,001). Estos resulthdos indican que no se escogió el mismo número de adjetivos de cada valencia y condición, y que además, la elección diferencial de dichos adjetivos dependió del grupo concreto.

Los análisis post-hoc (Duncan) entre los grupos mostraron que en la condición Actual, los pacientes con depresión mayor escogieron más rasgos negativos que los distímicos y los controles, y los distímicos más que los controles. Para los rasgos positivos se encontró el patrón inverso, escogiendo los controles mayor número que los pacientes con depresión mayor y los distímicos, y éstos más que los pacientes con depresión mayor. En cuanto a las condiciones Pasado y Futuro, se encontraron las mismas diferencias entre los grupos: Los pacientes deprimidos, pacientes con depresión mayor y distímicos, escogieron más autodescriptores negativos y menos positivos que los controles, y no aparecieron diferencias entre los dos grupos clínicos. Respecto a los rasgos neutros, no se encontraron diferencias entre los grupos para ninguna de las tres condiciones. En la Tabla 2 se presentan los valores medios (y DT) para estas variables.

\section{DISCUSIÓN}

Respecto al objetivo primero, pudimos constatar que durante un episodio depresivo los sujetos pueden distinguir claramente su estado actual de cómo eran en 
Tabla 2. Medias (DT) y post-hoc (Duncan) para la valencia y condición (presente, pasado y futuro) de los adjetivos escogidos en la tarea de codificación autorreferente

\begin{tabular}{|c|c|c|c|c|}
\hline Condición & Valencia & Grupo & Media $(D T)$ & Post-hoc \\
\hline \multirow{3}{*}{ ACTUAL } & Positivos & $\begin{array}{l}\mathrm{DM} \\
\mathrm{Di} \\
\mathrm{C}\end{array}$ & $\begin{array}{l}0,20(0,41) \\
2,50(2,43) \\
8,60(1,35)\end{array}$ & $\begin{array}{l}\text { DM - Di } \\
\text { DM - C } \\
\text { Di - C }\end{array}$ \\
\hline & Negativos & $\begin{array}{l}\mathrm{DM} \\
\mathrm{Di} \\
\mathrm{C}\end{array}$ & $\begin{array}{l}8,70(1,59) \\
7,38(2,52) \\
1,40(1,69)\end{array}$ & $\begin{array}{l}\mathrm{DM}-\mathrm{Di} \\
\mathrm{DM}-\mathrm{C} \\
\mathrm{Di}-\mathrm{C}\end{array}$ \\
\hline & Neutros & $\begin{array}{l}\mathrm{DM} \\
\mathrm{Di} \\
\mathrm{C}\end{array}$ & $\begin{array}{l}5,05(1,63) \\
5,77(1,62) \\
5,50(0,94)\end{array}$ & - \\
\hline \multirow{3}{*}{ PASADO } & Positivos & $\begin{array}{l}\mathrm{DM} \\
\mathrm{Di} \\
\mathrm{C}\end{array}$ & $\begin{array}{l}7,55(2,85) \\
6,72(3,57) \\
9,15(0,93)\end{array}$ & $\begin{array}{l}\text { DM - C } \\
\text { Di - C }\end{array}$ \\
\hline & Negativos & $\begin{array}{l}\mathrm{DM} \\
\mathrm{Di} \\
\mathrm{C}\end{array}$ & $\begin{array}{l}3,40(2,77) \\
3,77(3,17) \\
1,85(1,63)\end{array}$ & $\begin{array}{l}\mathrm{DM}-\mathrm{C} \\
\mathrm{Di}-\mathrm{C}\end{array}$ \\
\hline & Neutros & $\begin{array}{l}\mathrm{DM} \\
\mathrm{Di} \\
\mathrm{C}\end{array}$ & $\begin{array}{l}6,25(1,29) \\
5,50(1,68) \\
6,25(0,85)\end{array}$ & - \\
\hline \multirow{3}{*}{ FUTURO } & Positivos & $\begin{array}{l}\mathrm{DM} \\
\mathrm{Di} \\
\mathrm{C}\end{array}$ & $\begin{array}{l}6,10(3,86) \\
6,00(3,63) \\
9,15(1,87)\end{array}$ & $\begin{array}{l}\mathrm{DM}-\mathrm{C} \\
\mathrm{Di}-\mathrm{C}\end{array}$ \\
\hline & Negativos & $\begin{array}{l}\mathrm{DM} \\
\mathrm{Di} \\
\mathrm{C}\end{array}$ & $\begin{array}{l}4,45(3,26) \\
5,61(3,63) \\
1,60(1,87)\end{array}$ & $\begin{array}{l}\mathrm{DM}-\mathrm{C} \\
\mathrm{Di}-\mathrm{C}\end{array}$ \\
\hline & Neutros & $\begin{array}{l}\mathrm{DM} \\
\mathrm{Di} \\
\mathrm{C}\end{array}$ & $\begin{array}{l}6,00(1,12) \\
5,83(1,24) \\
6,05(0,75)\end{array}$ & - \\
\hline
\end{tabular}

Nota: $\mathrm{DM}=$ depresión mayor; $\mathrm{Di}=$ distimia, $\mathrm{C}=$ controles normales.

el pasado y cómo se esperan ver en el futuro. En concreto, ambos grupos clínicos se percibieron en el presente de forma predominantemente negativa, mientras que en el futuro se autoasdcribieron adjetivos positivos y negativos de forma proporcionada. Respecto al pasado, los distímicos también realizaron una autoasdcripción balanceada, y los pacientes con depresión mayor manifestaron una visión predominantemente positiva.

Ahora bien, si comparamos las autodescripciones de los sujetos para las tres condiciones temporales, los resultados anteriores se matizan de forma importante. Aunque ambos grupos clínicos manifestaron una expectativa de futuro menos negativa respecto a su estado actual, no llegaban a contemplarse «tan bien» como en el pasado. También resulta llamativo el hecho de que los pacientes con depresión mayor, pero no los distímicos, resalten la positividad de su pasado en relación a su estado actual y a su expectativa de futuro.

Estos resultados son congruentes con la sugerencia de Chaplin et al. (1988) respecto a que los individuos pueden organizar sus autodescriptores alrededor de conceptos de rasgo o estado. Y también coinciden con los encontrados por Brewin et al. (1992) acerca de que los deprimidos son capaces de distinguir su estado actual, predominantemente negativo, 
de cómo son, o creen ser, en general, condición en la que los autodescriptores positivos y negativos están más balanceados. Estos resultados pueden ayudar a entender las inconsistencias aparecidas en la literatura sobre los sesgos de memoria en deprimidos, que, supuestamente, potenciarían un mejor recuerdo de información negativa frente a la positiva o neutra. Se supone que lo que provoca estos sesgos hacia lo negativo es la existencia de una correspondencia entre la valencia (o el contenido) de los adjetivos que se utilizan como estímulos y los contenidos - negativos - de las autodescripciones que se hallan activas en las representaciones mentales de los pacientes (i. e. «esquemas depresógenos», Beck, 1976; "nodo de la depresión», Bower, 1981). Nuestros resultados indican que cuando pedimos a los deprimidos que se autodescriban (que elijan) en el momento actual, escogen preferentemente descriptores coherentes con su estado de ánimo negativo. Pero cuando se les pide que se autodescriban en el pasado y/o en el futuro, eligen además descriptores positivos, lo que indica que sí que tienen acceso a información positiva sobre sí mismos, pero que sólo informan sobre ella si se les pregunta adecuadamente. En consecuencia, es posible que según el material estimular que se le presente al paciente, o el contexto experimental utilizado, el procesamiento de información autorreferente esté más influido por su esquema activo en ese momento (i.e. negativo) o por representaciones más duraderas del Yo (de contenido positivo y negativo más balanceado). En el primer caso, la probabilidad de aparición de los sesgos de procesamiento antes mencionados sería mucho mayor que en el segundo caso.

Por otro lado, la dirección de las diferencias aparecidas en la autopercepción de los sujetos en los tres referentes temporales, son especialmente relevantes desde el punto de vista clínico. Nuestros resultados son coincidentes con algunos postulados de las principales teorías de la depresión. En concreto, con la Teoría Reformulada de la Indefensión Aprendida (Abramson et al., 1978) y el modelo cognitivo de Beck $(1967,1976)$, que enfatizan la importancia de la desesperanza sobre el futuro en la etiología, mantenimiento y tratamiento de la depresión.

Ahora bien, los sujetos de nuestro estudio no mostraron una visión indiscriminadamente catastrofista acerca de su futuro. Presumían una mejoría respecto a su estado actual, aspecto que quizá les inducía a buscar tratamiento y a mantenerlo, pero a la vez, asumían que dicha mejoría no les devolvería a su estado de bienestar previo a la depresión. Este aspecto era además más evidente en los pacientes con depresión mayor, que mostraron una visión de su pasado más marcadamente positiva que los distímicos. Esto último discreparía con los datos aportados por algunos autores (Gibb et al., 2001) que asocian la vulnerabilidad cognitiva para la depresión con una historia personal traumática (i.e. maltrato emocional infantil). Estas cuestiones trascienden los objetivos de este estudio, pero parece clara la necesidad de efectuar estudios longitudinales que evalúen, junto a otros factores, la autopercepción de los sujetos antes de padecer el trastorno y una vez remitido el mismo, y comprobar de ese modo si en efecto la depresión se asocia a una historia personal traumática y si deja un poso experiencial que supone un punto de inflexión en la vida de los sujetos que lo padecen. En cualquier caso, desde el punto de vista clínico, lo reseñable en nuestro estudio es la constatación de una visión negativa respecto al futuro en estos sujetos en el momento en que están padeciendo un trastorno depresivo, aspecto ineludible en el proceso terapéutico por sus implicaciones sobre conductas suicidas, abandonos de la terapia e inducción a las recaídas. 
Los resultados obtenidos para el segundo objetivo de nuestro estudio vienen a completar los anteriores. Observamos que los deprimidos, con depresión mayor o distimia, muestran una visión de sí mismos en la actualidad diferente (más negativa y menos positiva) a la de los no deprimidos. Sin embargo, no se evidencia que su percepción del pasado y su expectativa de futuro sea idéntica en los tres grupos. Ambos grupos clínicos manifiestan mayor negatividad y menor positividad que el grupo control tanto para su futuro como para su pasado. Respecto a su visión de futuro, estos datos son coincidentes con los encontrados por Garber y Hollon (1980) y Krantz y Hammen (1979) que revelan que en comparación con los no deprimidos, los sujetos deprimidos proporcionan predicciones pesimistas sobre el futuro. En cuanto a la autopercepción de su pasado, en comparación con los controles, se pueden apuntar distintas explicaciones: En primer lugar, cabría pensar que la valoración más negativa de los deprimidos acerca de su pasado se debe, efectivamente, a la existencia de una historia personal traumática. Este aspecto está pendiente de verificar, pero en cualquier caso, no sería coherente con el resultado obtenido para el objetivo primero (intra grupo - entre condiciones) que indica que los deprimidos realizan una autoevaluación marcadamente positiva de su pasado en comparación con su presente y su futuro. En segundo lugar, se podría considerar que la presencia actual de la depresión produce un sesgo negativo general que impregna las autoevaluaciones del pasado, además de las del presente. Aunque, teniendo en cuenta que las diferencias (valoración negativa del pasado) aparecen sólo al comparar los deprimidos con los controles, ¿sería posible que fueran los sujetos no deprimidos los que presentan un sesgo positivo en sus autopercepciones, tanto actuales como de su pasado?. La evidencia de un autoesquema marcadamente positivo en los sujetos no deprimidos se constata en numerosos estudios (Abramson y Alloy, 1981; Greenberg et al., 1988). Asimismo, la evidencia de sesgos positivos en el procesamiento de información para los sujetos no deprimidos, también se ha mostrado consistente en muchas investigaciones (Derry y Kuiper, 1981; Greenberg et al., 1988; Hedlund y Rude, 1995; Calev, 1996). Calev (1996) se refiere a este efecto como la «tendencia pollyana». En definitiva, para poder profundizar en estas explicaciones, se hace imprescindible continuar la investigación con nuevos estudios controlados.

En resumen, se observó que los deprimidos tenían una visión sobre su pasado y su futuro compuesta de elementos positivos y negativos de forma proporcionada. Y por último, constatamos que en el futuro estos sujetos se percibían mejor que en el presente, pero no tan bien como en el pasado. $Y$ con los resultados derivados del objetivo segundo, observamos que además de en el presente, los deprimidos se perciben de forma más negativa y menos positiva que los no deprimidos en el pasado y en el futuro.

En definitiva, si nos situamos dentro de la esfera de la depresión, vemos que los sujetos deprimidos efectivamente organizan sus autodescriptores alrededor de marcadores temporales. $Y$ vemos también que pueden utilizar "escalas de grises» para verse a sí mismos según el referente temporal del que partan. Ahora bien, es al compararlos con los no deprimidos cuando estos sujetos utilizan únicamente el color "negro" para describirse a sí mismos, independientemente del referente temporal que utilicen.

Desde el punto de vista clínico es importante reconocer y utilizar los distintos matices que proporciona la autopercepción depresiva, y no dejar que el marcado y global contraste con la auto- 
percepción no depresiva nos remita a la creencia popular de que el deprimido es aquel individuo que todo lo ve «negro».

\section{REFERENCIAS BIBLIOGRÁFICAS}

Abramson, L.Y., Seligman, M.E.P. y Teasdale, J. (1978). Learned helplessness in humans: Critique and reformulation. Journal of Abnormal Psychology, 87, 49-74.

Abramson, L.Y., y Alloy, L.B. (1981). Depression, nondepression, and cognitive illusions: reply to Schwartz. Journal of EXperimental Psychology: General, 110, 436-447

American Psychiatric Associaton (1994). Diagnostic and Statistical manual of Mental Disorders ( $4^{\text {th }}$ ed.). Washington, DC, APA (Barcelona: Masson, 1995).

Beck, A.T. (1967). Depression: Clinical, Experimental, and Theoretical Aspects. New York: Harper \& Row.

Beck, A.T. (1976). Cognitive therapy and the emotional disorders. New York: International University Press.

Beck, A.T., y Clark, D.A. (1988). Anxiety and depression: An information processing perspective. Anxiety Research, 1, 23-26.

Beck, A.T., Rush, A.J., Shaw, B.F., y Emery, R.A. (1979). Cognitive therapy of depresion. New York, Guilford Press.

Bower, G.H. (1981). Mood and memory. American Psychologist, 36, 129-148.

Bradley, B., y Mathews, A. (1983). Negative self-schemata in clinical depression. British Journal of Clinical Psychology, 22, 173-182.

Brewin, C.R., Smith, A.J., Power, M. y Furnham, A. (1992). State and trait differences in depressive self-perceptions. Behavior Research and Therapy, 30, 555-557.

Brown, G., Andrews, B., Bifulco, A., y Veiel, H. (1990). Self-esteem and depression. I. Measurement issues and prediction of onset. Social Psychiatry Epidemiology, 25, 200-209.

Calev, A. (1996). Affect and memory in depression: Evidence of better delayed recall of positive than negative affect words. Psychopathology, 29, 71-76.
Chaplin, W.F., John, O.P., y Goldberg, L.R. (1988). Conceptions of states and traits: Dimensional attributes with ideas as prototypes. Journal of Personality and Social Psychology, 54, 541-557.

Clark, D.A., y Steer, R.A. (1996). Empirical status of the cognitive model of anxiety and depression. En P. M. Salkovskis (Ed.) Frontiers of Cognitive Therapy (págs. 7596). New York: Guilford Press

Clark, D.A., Beck, A.T., y Alford, B.A. (1999). Scientific foundations of cognitive theory and therapy of depression. Philadelphia: Wiley.

Clark, D.M., y Teasdale, J.D. (1985). Constraints of the effects of mood on memory. Journal of Personality and Social Psychology, 48, 1595-1608.

Craik, F.I.M. y Lockhart, R.S. (1972). Levels of processing: A framework for memory research. Journal of Verbal Learning and Verbal behavior, 11, 671-684

Derry, P.A., y Kuiper, N.A. (1981). Schematic processing and self-reference in clinical depression. Journal of Abnormal Psychology, 90, 286-297.

Dobson, K.S., y Shaw, B.F. (1987). The specificity and stability of self-referential encoding in clinical depression. Journal of Abnormal Psychology, 96, 34-40.

Dohr, K.B., Rush, A.J., y Bernstein, I.H. (1989). Cognitive biases and depression. Journal of Abnormal Psychology, 98, 263267

Dozois, D.J.A., y Dobson, K.S. (2001). Information Processing and Cognitive Organization in Unipolar Depression: Specificity and Comorbidity Issues. Journal of Abnormal Psychology, 110, 236-246

First, M.B., Spitzer, R.L., Gibbon, M., y Williams, J.B. (1997). Structured Clinical Interview for DSM IV Axis I Disorders (SCID-I), Clinical Version: Administration Booklet. Washington: American Psychiatric. Press Inc.

Garber, J., y Hollon, S.D. (1980) Universal versus personal helplessness in depression: Belief in uncontrolability or incompetence?. Journal of Abnormal Psychology, 89, 56-66

Gibb, B.E., Alloy, L.B., Abramson, L.Y., Rose, D.T., Whitehouse, W.G., Donovan, P., 
Hogan, M.E., Cronholm, J., y Tierney, S. (2001). History of childhood maltreatment, negative cognitive styles, and episodes of depression in adulthood. Cognitive Therapy and Research, 25, 425-446

Giménez, A. (2000). Depresión y procesamiento de información: El autoesquema y la codificación de información autorreferente. Tesis Doctoral. Universidad de Valencia, Facultad de Psicología.

Gotlib, I.M., y Mc Cann, C.A. (1984). Construct accesibility and depression: An examination of cognitive and affective factors. Journal of Personality and Social Psychology, 47, 427-439.

Greenberg, M.S., Vázquez, C.V., y Alloy, L.B. (1988). Depression versus anxiety: Differences in Self -and Other- schemata. En L.B. Alloy (Ed.). Cognitive Processes in Depression (pp.109-142). New York: Guilford Press

Haaga, D.A., Dyck, M.J., y Ernst, D. (1991). Empirical status of cognitive theory of depression. Psychological Bulletin, 2, 215236

Hammen, C., Marks, T., De Mayo, R., y Mayol, A. (1985). Self-schemas and risk for depression: A prospective study. Journal of Personality and Social psychology, 49, 1147-1159

Hedlund, S., y Rude, S.S. (1995). Evidence of latent depressive schemas in formerly depressed individuals. Journal of Abnormal Psychology, 3, 517-525.

Ingram, R.E.(1984). Toward and information processing analysis of depression. Cognitive Therapy and Research, 8, 443-478.

Ingram, R.E., y Reed, M.J. (1986). Information encoding and retrieval processes in depresión: Findings, issues, and future directions. En R.E. Ingram (Ed.), Information Processing Approaches to Clinical Psychology (pp. 131-150). Orlando: Academic Press.

Ingram, R.E., Partridge, S., Scott, W., y Bernet, C. (1994). Schema specificity in Subclinical Syndrome Depression: Distinctions between automatically versus effortfully encoded state and trait depressive information. Cognitive Therapy and Research, 18, 195209.

Ingram, R.E., Fidaleo, R.A., Friedberg, R.,
Shenk, J.L., y Bernet, C.Z. (1995). Content and mode of information processing in Major Depressive Disorder. Cognitive Therapy and Research, 19, 281-293.

Ingram, R.E., Miranda, J., y Segal, Z.V. (1998). Cognitive vulnerability to depression. New York: Guilford Press.

Krantz, S., y Hammen, C.L. (1979). Assessment of cognitive bias in depression. Journal of Abnormal Psychology, 88, 611-619

Kuiper, N.A., y MacDonald, M.R. (1982). Self and other perception in mild depressives. Social Cognition, 1, 223-229.

Kuiper, N.A., y Olinger, L.J. (1986). Dysfunctional attitudes and self-worth contingency model of depression. Advances in Cognitive-Behavioural Research and Therapy, 5, 115-142.

Kuiper, N.A., Olinger, L.J., y MacDonald, M.R. (1988). Vulnerability and episodic cognitions in a self-worth contingency model of depression. En L.B. Alloy (Ed), Cognitive processes in depression. (pp. 289-309). New York: Guilford.

Matt, G.E., Vázquez, C. y Campbell, W.K. (1992). Mood-congruent recall of affectively toned stimuli. A meta-analytic review. Clinical Psychology Review, 12, 227-256.

McLeod, C., y Hagan, R. (1992). Individual differences in the selective processing of threatening information, and emotional responses to a stressful life event. Behaviour Research and Therapy, 30,151-161

Power, M.J. (1987). Cognitive failures, dysfunctional attitudes, and symptomatology: A longitudinal study. Cognition and Emotion, 2, 133-143

Ruipérez, M.A. (1994). Contenido del autoesquema, sesgos de memoria y de atención en pacientres deprimidos versus ansiosos: $U n$ estudio experimental. Tesis Doctoral. Universidad de Valencia, Facultad de Psicología.

Ruiz, J.A. (1989). Estado de ánimo y procesamiento de información emocionalmente congruente: ¿Sesgo selectivo?. En B. Sandin, y J. Bermúdez (Eds.), Procesos emocionales y salud. (pp. 141-168). Madrid: UNED.

Salkovskis, P.M. (Ed.) (1996). Frontiers of cognitive therapy. New York: Guilford Press.

Segal, Z.V., y Swallow, S.R. (1994). Cognitive assessment of unipolar depression: Measu- 
ring products, processes and structures. Behavior Research and Therapy, 32,147-158

Segal, Z.V., y Gemar, M. (1997). Changes in cognitive organisation for negative self-referent material following cognitive behaviour therapy for depression: A primed Stroop study. Cognition and Emotion, 11, 501-516
Teasdale, J.D. (1983). Negative thinking in depression: Cause, effect or reciprocal relationship?. Advances in Behavioral Research and Therapy, 5, 3-25

Teasdale, J.D. (1988). Cognitive vulnerability to persistent depression. Cognition and Emotion, 2, 247-274

Anexo 1. Estímulos verbales utilizados para la tarea de codificación autorreferente

\begin{tabular}{lll}
\hline & \multicolumn{2}{c}{ Estados de ánimo } \\
\hline Positivos & Negativos & $\begin{array}{l}\text { Rasgos de personalidad neutros en } \\
\text { relación con estados de ánimo }\end{array}$ \\
\hline Alegre & Preocupado & Presumido \\
Dichoso & Triste & Cobarde \\
Satisfecho & Infeliz & Inseguro \\
Risueño & Lloroso & Sano \\
Feliz & Desdichado & Comprensivo \\
Optimista & Sombrío & Pacífico \\
Juerguista & Melancólico & Sereno \\
Contento & Fracasado & Agradable \\
Divertido & Angustiado & Rígido \\
Animado & Apenado & Agresivo \\
\hline
\end{tabular}

